\title{
Phase-space tomography with a programmable Radon-Wigner display
}

\author{
Alejandro Cámara, ${ }^{1, *}$ Tatiana Alieva, ${ }^{1}$ José A. Rodrigo, ${ }^{2}$ and Mariá L. Calvo ${ }^{1}$ \\ ${ }^{1}$ Universidad Complutense de Madrid, Facultad de Ciencias Físicas, Ciudad Universitaria s/n, 28040 Madrid, Spain \\ ${ }^{2}$ Instituto de Óptica, Consejo Superior de Investigaciones Cientificas, Serrano 121, 28006 Madrid, Spain \\ *Corresponding author: a.camara@fis.ucm.es
}

Received April 15, 2011; revised May 25, 2011; accepted May 26, 2011; posted May 27, 2011 (Doc. ID 146047); published June 21, 2011

\begin{abstract}
We show the adaptation of a multifunctional optical system consisting of two spatial light modulators for the optimal measurement of the Radon-Wigner transform of one-dimensional signals. The proposed Radon-Wigner display allows reconstructing the Wigner distribution and the phase or the mutual intensity of fully or partially coherent fields, respectively. It is also suitable for the analysis of two-dimensional rotationally symmetric or separable in Cartesian coordinates optical fields. The feasibility of the proposed scheme is experimentally demonstrated in several examples. @ 2011 Optical Society of America

OCIS codes: $\quad 030.0030,070.0070,090.1760,050.5082,100.5070,080.5084$.
\end{abstract}

The Wigner distribution (WD) plays an important role in analysis and synthesis of different types of signals, including fully and partially coherent optical fields. The set of the WD projections at an arbitrary angle in phase-space, known as a Radon-Wigner transform (RWT) [1], contains the same information about the signal as the $\overline{\mathrm{WD}}$. In contrast to the WD, the RWT takes only nonnegative values and, therefore, can be directly measured [2]. We regard that the fractional Fourier transform (FT) yields to the rotation of the WD in phase-space, while the fractional power spectra coincide with the WD projections.

In the case of one-dimensional (1D) signals, the RWT is a two-dimensional (2D) function defined as

$P(\alpha, x)=\int \mathrm{d} q W\left(x \cos \alpha-s^{2} q \sin \alpha, q \cos \alpha+s^{-2} x \sin \alpha\right)$,

where $W(x, q)=\int \mathrm{d} x^{\prime} \Gamma\left(x+x^{\prime} / 2, x-x^{\prime} / 2\right) \exp \left(-i 2 \pi x^{\prime} q\right)$ is the WD of the signal, $(x, q)$ are the space and frequency coordinates, $\Gamma\left(x_{1}, x_{2}\right)=\left\langle f\left(x_{1}\right) f^{*}\left(x_{2}\right)\right\rangle$ is the mutual intensity of the signal $f(\cdot)$, and $s$ is a normalization parameter with dimension of length that depends on the system implementation. The transformation angle $\alpha$ varies in a $\pi$ interval. The kernel of the fractional FT needed for the acquisition of the corresponding projections, $P(\alpha, x)=\left\langle\left|\int \mathrm{d} u f(u) K^{\alpha}(x, u)\right|^{2}\right\rangle$, is given by

$$
K^{\alpha}(x, u)=\frac{\exp \left\{\frac{i \pi}{s^{2} \sin \alpha}\left[\left(x^{2}+u^{2}\right) \cos \alpha-2 x u\right]\right\}}{s \sqrt{i \sin \alpha}} .
$$

Several schemes for the RWT visualization-RadonWigner displays (RWDs)-have been proposed. They are based on the use of either combinations of Fresnel zone plates [3] or varifocal glass lenses [4,5]. In spite of important achievements, these setups have not found wide application in signal processing due to their complex design and the involvement of nonconventional optical elements. Moreover, in most of the schemes, each projection has a different magnification depending on the angle $\alpha$.

0146-9592/11/132441-03\$15.00/0
The use of spatial light modulators (SLMs) allows constructing an RWD that avoids the previous inconveniences and provides flexibility to the system. Here, we demonstrate how a multifunctional optical scheme consisting of two SLMs can be easily converted into an RWD. Its performance is verified experimentally. We also show that the RWD, primarily created for the description of 1D signals, can also be applied for the analysis of $2 \mathrm{D}$ optical fields.

A recently proposed optical system for measuring the 2D fractional FT power spectra for arbitrary angles $\left(\alpha_{x}, \alpha_{y}\right)$ in the interval $[\pi / 2,3 \pi / 2]$ without magnification depending on the angle is reported in [6]. It consists of two generalized lenses implemented by reflective SLMs working in phase-only modulation and a CCD camera used for the spectra registration. The optical path length $z$ between these three elements is fixed. Each generalized lens is a combination of two orthogonally crossed cylindrical lenses whose powers depend on the transformation angles. The change of the parameters $\left(\alpha_{x}, \alpha_{y}\right)$ is produced almost in real time by the modification of the digital lens power. Note that the same optical setup is suitable for other tasks, including 2D signal filtering, correlation and wavelet performance, gyrator transformation, mode conversion, etc. [7].

This setup can be easily adapted for single-shot registration of the RWT of 1D signals (see Fig. 1). We suppose that the $x$-dependent $1 \mathrm{D}$ signal is first converted into a $2 \mathrm{D}$ one, which is its multiple-copy along the $y$-axis, by the use of a conventional cylindrical lens arrangement (e.g. see Fig. 1 of []]). Then, it is projected onto the first SLM. Each SLM encodes a varifocal lens with phase modulation in the $j$ th row given by [ $[6]$

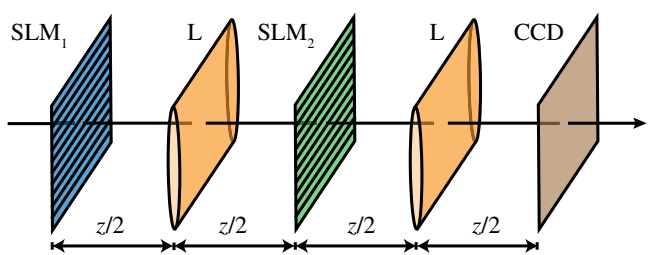

Fig. 1. (Color online) Radon-Wigner display scheme. 


$$
\begin{gathered}
t^{(1)}(x ; j)=\exp \left[-\frac{i \pi}{s^{2}} x^{2}\left(2-\cot \frac{\alpha_{j}}{2}\right)\right], \\
t^{(2)}(x ; j)=\exp \left[-\frac{i \pi}{s^{2}} x^{2} 4\left(1-\sin \alpha_{N-j+1}\right)\right],
\end{gathered}
$$

where $j=1, \ldots, N, N$ is the number of rows of the SLM, $\alpha_{j}=\pi / 2+(j-1) \pi / N$ is the transformation angle associated with the $j$ th channel, $s=\sqrt{2 \lambda z}$ is the system normalization parameter, and $\lambda$ is the wavelength. To compensate for the diffraction and match the channels between the SLMs and the CCD, two identical convergent cylindrical lenses (L in Fig. 1) of power $4 / z$ operating along the $y$-axis are placed at a distance $z / 2$ from each SLM. The CCD camera captures the image associated with the $N$-channel RWT of the $1 \mathrm{D}$ signal.

Note that all registered WD projections have the same magnification and, therefore, can be used for signal analysis without additional post processing. Moreover, the use of SLMs allows for increasing the number of channels $(m N)$, and, hence, the precision of the measurement, if the RWT is captured in $m$ shots. For example, the concatenation of the $N$ WD projections obtained for angles in the region $\alpha \in(\pi / 2, \pi]$ and $\alpha \in(\pi, 3 \pi / 2]$ acquired in two independent registrations leads to the $2 N$-channel RWT.

Let us now discuss the experimental realization and application of the RWD presented in Fig. 1. The collimated beam from an Nd:YAG laser $(\lambda=532 \mathrm{~nm})$ is projected onto the first SLM. In order to avoid the conversion of the $1 \mathrm{D}$ signal into the $2 \mathrm{D}$ one, we implement this last one together with the corresponding digital varifocal lens $t^{(1)}$ by the first SLM. This field amplitude is encoded by a phase-only hologram synthesized using the method proposed in [9]. In each reflective SLM (Holoeye LCR-2500, pixel pitch of $19 \mu \mathrm{m}$ and 1024 pixels $\times$ 768 pixels), $N=293$ channels are addressed. The optical path length between the SLMs is $z=1 \mathrm{~m}$, leading to $s=0.1 \mathrm{~mm}$. The output intensity is registered by a CCD camera (Spiricon SP620U, 12 bit gray level, pixel pitch of $4.4 \mu \mathrm{m}$ and 1600 pixels $\times 1200$ pixels) placed at $z=1 \mathrm{~m}$ from the second SLM.

To demonstrate the feasibility of the experimental setup, a window $\operatorname{signal} \operatorname{rect}(x / w)$ of width $w=2.47 \mathrm{~mm}$, yielding a WD of rather fine structure, has been considered. The WD is reconstructed from the RWT using the inverse Radon transform. A good agreement between the RWTs and the WDs obtained experimentally [see Figs. 2(a) and 2(b)] and simulated numerically [see Fig. 2(c) and 2(d)] is observed.

Measuring the RWT allows the identification of the quadratic chirp components of signals [1] , which is particularly relevant for the characterization of optical systems. The angle $\alpha$ corresponding to the intensity peak in the RWT indicates that the chirp $\exp \left[i \pi x^{2} /\left(\lambda f_{c}\right)\right]$ has parameter $f_{c}=-s^{2} \lambda^{-1} \tan \alpha$. Another method of chirp characterization is the estimation of the angle between the distinctive line structure of its WD and the $x$-axis. The RWT and the reconstructed WD of the windowed $\operatorname{chirp} \operatorname{rect}(x / w) \exp \left[i \pi x^{2} /\left(\lambda f_{c}\right)\right]$, with $w=2.47 \mathrm{~mm}$ and $f_{c}=0.93 \mathrm{~m}$, are displayed in Figs. 3(a) and 3(b), respectively. The WD obtained from double-shot $(\overline{m=})$ RWT registration shown in Fig. 3(c) confirms the improvement
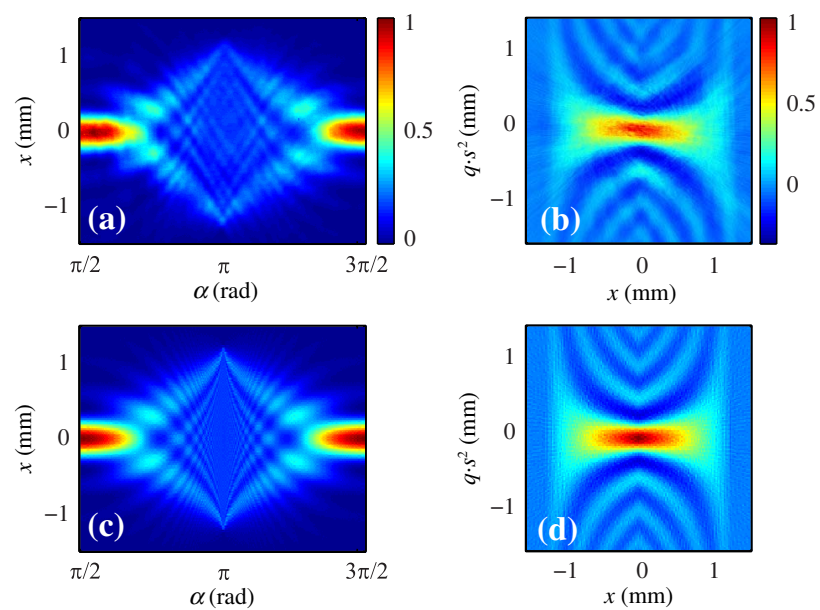

Fig. 2. (Color online) (a) Experimental and (c) numerically simulated RWT of a window signal. WD reconstructed from the (b) experimental and (d) simulated RWT.

of measurement precision by increasing the number of channels. In particular, the fine interferometric fringes of the WD are clearly observed. The values of the chirp parameter estimated from the RWT registered by singleand double-shot measurements are $f_{c}=1.09 \mathrm{~m}$ and $f_{c}=0.91 \mathrm{~m}$, respectively, and from the corresponding WDs are $f_{c}=1.15 \mathrm{~m}$ and $f_{c}=0.95 \mathrm{~m}$.

Since the mutual intensity corresponds to the FT of the $\mathrm{WD}$, knowledge of the RWT or the WD is also very important for the analysis of the beam coherence properties. In the case of coherent signals, i.e., $\Gamma\left(x_{1}, x_{2}\right)=$ $f\left(x_{1}\right) f^{*}\left(x_{2}\right)$, its phase, apart from a constant, can be retrieved when one variable $x_{2}$ is fixed and $f\left(x_{2}\right) \neq 0$. A successful phase recovery is demonstrated in Fig. $\underline{4}$ for the case of single- and double-shot RWT registration of the studied chirp signal.

The RWD can also be used for the analysis of $2 \mathrm{D}$ optical fields. It has been shown in [8] that the fourdimensional WD of rotationally symmetric beams can be reconstructed from the $\mathrm{WD}$ of the $1 \mathrm{D}$ field corresponding
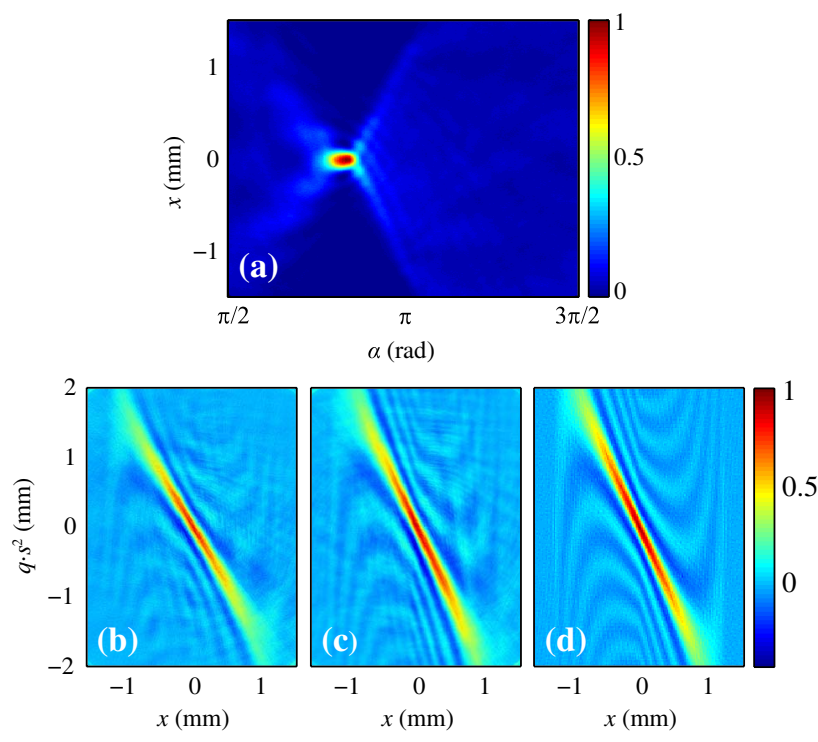

Fig. 3. (Color online) (a) Experimental RWT of a windowed chirp signal and its WD recovered from (b) single- and (c) double-shot measurements. (d) Simulated WD. 


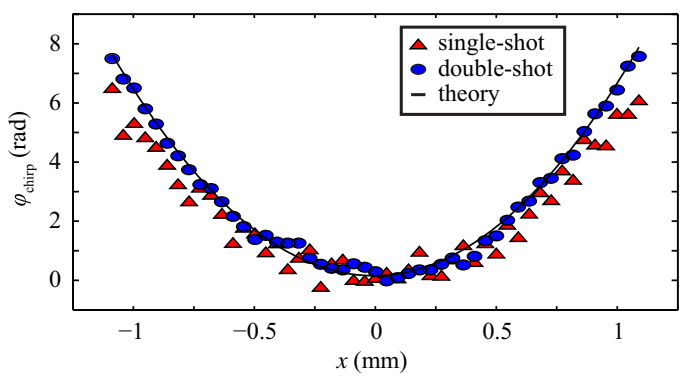

Fig. 4. (Color online) Theoretical (solid black line) and experimental phase profiles retrieved from single- and double-shot measurements of the RWT of a windowed chirp signal with parameter $f_{c}=0.93 \mathrm{~m}$.

to its radial profile in a transversal plane. The required 1D signal is obtained by placing across the beam a fine slit aperture along the $x$-axis. This signal is further converted into a $2 \mathrm{D} x$-dependent one. As an example, we have considered the rotational symmetric beam $\mathrm{LG}_{0,0}+2 \mathrm{LG}_{2,0}$, where $\mathrm{LG}_{p, l}$ is the helical Laguerre-Gaussian mode with radial $p$ and azimuthal $l$ indices. Note that this beam is reciprocal under the FT and, therefore, its original form is recovered in the far field. Furthermore, its transversal intensity distribution transforms from a central spot into the doughnutlike structure and vice versa during the propagation. In Figs. 5(a) and 5(b), the experimental and simulated WD of its radial profile are shown. Based on this data, the four-dimensional WD of the entire beam can be reconstructed.

The WD of 2D fields separable into Cartesian coordinates, for example the Hermite-Gaussian (HG) beams, can also be reconstructed by applying the RWD. The mutual intensity and the WD of such beams are also separable [10]: $W(\mathbf{r}, \mathbf{q})=W_{x}\left(x, q_{x}\right) W_{y}\left(y, q_{y}\right)$. The transformation of such beams into two 1D ones is achieved by a system consisting of two cylindrical lenses that performs the FT and imaging operation in the $y$ and $x$ directions, respectively. Thus, the output field amplitude has the form $F(x, u)=\int \mathrm{d} y f(x, y) \exp (-i 2 \pi y u)$, which is converted into the $1 \mathrm{D}$ signal proportional to $f(x)$ for $u=0$. To select $f(x)$, we apply again a fine slit aperture along the $x$-axis. In order to reconstruct the WD of both 1D signals, either the input beam or the cylindrical lenses of the setup, including the digital lenses (but not the SLMs), have to be rotated at $\pi / 2$. The Cartesian product of the WDs corresponds to the four-dimensional WD of the 2D field. In Figs. 5(c) and 5(d), the experimental and simulated WDs for the $1 \mathrm{D} \mathrm{HG}$ signal $\mathrm{HG}_{4}$ are compared.

The multifunctionality and flexibility of the proposed RWD, along with the good agreement between the experimental results and the numerical simulations, make the system a versatile tool for characterization
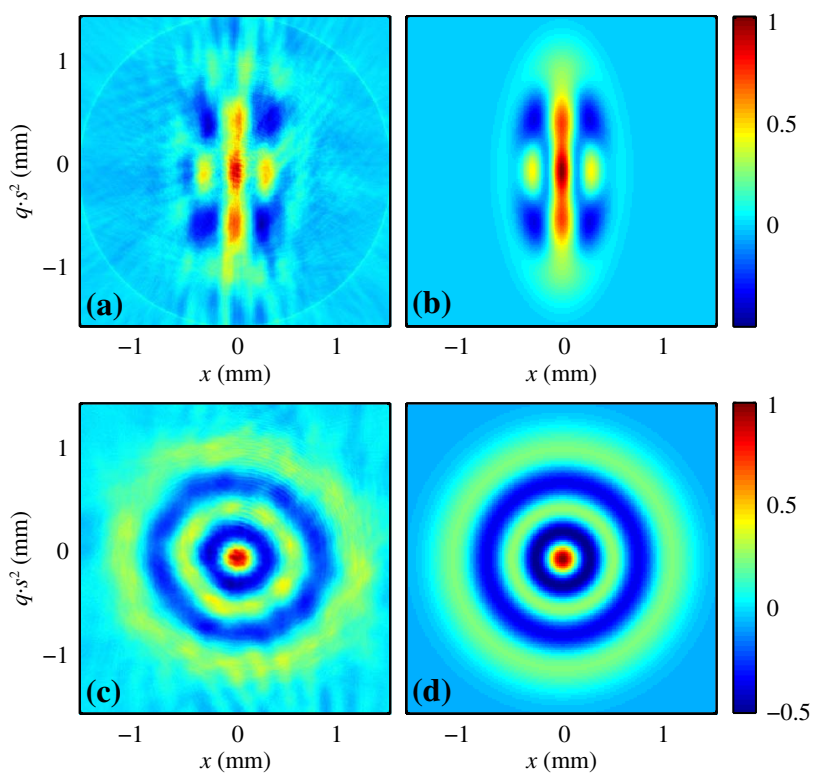

Fig. 5. (Color online) (a), (c) Experimental and (b), (d) simulated WD of the radial profile of the $\mathrm{LG}_{0,0}+2 \mathrm{LG}_{2,0}$ beam and of the $\mathrm{HG}_{4}$ signal, respectively.

of $1 \mathrm{D}$ and 2D optical fields of different degrees of spatial coherence.

The project TEC2008-04105 from the Spanish Ministry of Science and Innovation is acknowledged. A. Cámara and J. A. Rodrigo thank the financial support from the Consejería de Educación de la Comunidad de Madrid together with the European Social Fund and Juan de la Cierva grant.

\section{References}

1. J. Wood and D. Barry, IEEE Trans. Signal Process. 42, 2105 (1994).

2. M. G. Raymer, M. Beck, and D. F. McAlister, Phys. Rev. Lett. 72, 1137 (1994).

3. D. Mendlovic, R. G. Dorsch, A. W. Lohmann, Z. Zalevsky, and C. Ferreira, Appl. Opt. 35, 3925 (1996).

4. Y. Zhang, B.-Y. Gu, B.-Z. Dong, and G.-Z. Yang, Opt. Lett. 23, 1126 (1998).

5. W. D. Furlan, C. Soriano, and G. Saavedra, Appl. Opt. 47, E63 (2008).

6. J. A. Rodrigo, T. Alieva, and M. L. Calvo, Opt. Express 17, 4976 (2009).

7. J. A. Rodrigo, T. Alieva, and M. J. Bastiaans, in Optical and Digital Image Processing, G. Cristóbal, P. Schelkens, and H. Thienpont, eds. (Wiley, 2011), pp. 251.

8. G. S. Agarwal and R. Simon, Opt. Lett. 25, 1379 (2000).

9. J. Davis, D. Cottrell, J. Campos, M. Yzuel, and I. Moreno, Appl. Opt. 38, 5004 (1999).

10. A. Cámara, T. Alieva, J. A. Rodrigo, and M. L. Calvo, J. Opt. Soc. Am. A 26, 1301 (2009). 